\title{
Política Agraria Común y la de cohesión Frente a la Estrategia Europa 2020
}

\author{
Antonio González Temprano*
}

Fecha de recepción: 27 de agosto de 2012. Fecha de aceptación: 17 de enero de 2013.

\begin{abstract}
RESUMEN
El presente trabajo analiza la evolución más reciente de las dos grandes políticas de la uE, la Política Agraria Común y la política de cohesión, así como sus reformas para adecuarse a los objetivos de la Estrategia Europa 2020. El centro de esta investigación es el Presupuesto de gasto durante el Marco Financiero 2007-2013 y el rol que está ejerciendo en la consecución de una Europa más armónica, sectorial y territorialmente, objetivo vertebrador de la uE desde su fundación. La conclusión es que durante la primera mitad de este Marco Financiero se ha seguido progresando en una Unión más cohesionada sectorialmente, pero se ha debilitado la política de convergencia territorial respecto de la UE15, pese a que la dispersión de renta es mucho más acusada en la UE27 por las ampliaciones de 2004 y 2007; los doce países incorporados en estas fechas han sido, fundamental y paradójicamente, los afectados por tal debilitamiento.
\end{abstract}

Palabras clave: Estrategia Europa 2020, gasto, PAC, política de cohesión, Unión Europea.

\section{Common Agricultural and Cohesion Policy IN THE EUrope 2020 Strategy}

\begin{abstract}
This work analyzes the most recent evolution of the Eu's two major policies, the Common Agricultural Policy and Cohesion Policy, as well as the reforms made to adjust these policies to match the goals of the Europe 2020 Strategy. This research focuses on the expense budget of the Financial Framework 2006-2013, and the role it is playing in achieving a more harmonious Europe in sector and territorial terms, which has been the backbone objective of the EU since its founding. The work concludes that during the first half of this Financial Framework, the Union continued to progress to greater sector cohesion, but territorial convergence policies related to the eu 15 have been weakened, despite the fact that income dispersion has been multiplied due to expansions in 2004 and 2007. Paradoxically, the twelve countries incorporated in those years have been fundamentally affected by this weakening.
\end{abstract}

Key Words: Europe 2020 Strategy, Expenses, CAP, Cohesion Policy, European Union.

* Investigador de la Universidad Complutense de Madrid. Correo electrónico: temprano@cps.ucm.es 


\section{POLITIQUE AGRICOLE COMMUNE ET CELLE DE COHÉSION FACE À LA STRATÉGIE EUROPE 2020 \\ Résumé}

Le présent travail analyse l'évolution la plus récente des deux grandes politiques de l'ue, la Politique Agricole Commune et la politique de cohésion, ainsi que les réformes appliquées à celles-ci pour les adapter aux objectifs de Stratégie Europe 2020. L'axe de cette recherche est le Budget des dépenses durant le Cadre Financier 2007-2013 et son rôle dans l'obtention d'une Europe plus harmonique aux niveaux sectoriel et territorial, objectif qui structure l'ue depuis sa fondation. La conclusion est que durant la première moitié de ce Cadre Financier, il a continué à y avoir une avancée vers une Union ayant plus de cohésion au niveau sectoriel, mais la politique de convergence territoriale a été affaiblie par rapport à l'Europe des 15 , bien que se soit multipliée la dispersion de la rente du fait des extensions de 2004 et 2007 ; les douze pays incorporés ont été, de façon fondamentale et paradoxale, ceux qui ont été affectés par un tel affaiblissement.

Mots clés: Stratégie l'Europe 2020, dépense, PAC, politique de cohésion, l'Union Européenne.

\section{POLITICA AGRÁRIA COMUM E A COESÃO FRENTE À ESTRATÉGIA EUROPA 2020 Resumo}

O presente trabalho analisa a evoluçáo mais recente das dois grandes politicas da UE, a Politica Agrária Comum e a política de coesão social, assim como suas reformas para se adequar aos objetivos da Estratégia Europa 2020. O centro desta pesquisa é o Orçamento de gatos durante o Marco Financeiro 2007-2013 e o papel que esta exercendo na construção de uma Europa mais harmônica, setorial e territorialmente; objetivo vertebral da Eu desde a sua fundação. A conclusão é que durante a primeira metade de este Marco Financeiro se seguiu progredindo numa União mais entrelaçada setorialmente, mas se debilitou a politica de convergência territorial da UE15, apesar de se ter multiplicado a dispersão da renda pelas ampliaçôes de 2004 e 2007; os doze países incorporados nestas foram, fundamental e paradoxalmente, os afetados por tal enfraquecimento.

Palavras-chave: Estratégia Europa 2020, Gasto, PAC, Política de coesão, União Europeia.

\section{欧洲2020战略中的共同农业政策和凝聚政策}

摘要: 这项研究分析了欧盟共同农业政策和凝聚政策的最新进展以及相关政 策改革, 使之与欧洲2020战略目标相匹配。研究的重点是2006 2013年金 融框架的预算支出以及它在欧洲不同经济部门和国家实现更加和谐方面所扮 演的角色。自其成立之初, 该框架已成为欧盟的核心目标。研究结论认为, 在金融框架的第一部分, 欧盟继续实施行业凝聚政策, 但是欧盟十五个成 员国地区趋同政策被削弱了，尽管2004和2007年收入差距扩大了。矛盾的 是，有十二个国家在这两年因地区趋同政策被削弱都受到了影响。 关键词：欧洲2020年战略 支出 共同工业政策凝聚政策 欧盟 


\section{INTRODUCCIÓN}

La UE persigue, entre otros, dos grandes objetivos. Uno es la regulación de la actividad agraria, de la que se ocupa la Política Agraria Común (PAC, a partir de ahora), puesta en funcionamiento en 1962. El otro es la cohesión económica, social y territorial, un objetivo mucho más complejo y del que se encarga la política del mismo nombre, cuyo origen formal se sitúa en el Acta Única de 1986. ${ }^{1}$ Ambas políticas han estructurado el proyecto europeo durante el último cuarto de siglo.

La importancia de estas políticas se revela de muy diversas formas. Empecemos por la más nítida: su presencia en el Presupuesto de Gasto de la UE (Cuadro 1). En el ciclo plurianual actual, Marco Financiero 2007-2013, ambas políticas representan el $78 \%$ del Presupuesto, correspondiendo el $42 \%$ a la PAC y el 36\% a la política de cohesión. Con una peculiaridad: desde la entrada en vigor de los ciclos plurianuales (1988), el gasto de la PAC ha ido perdiendo peso y ganándolo la política de cohesión.

Otro factor que nos informa de la relevancia de estas dos políticas es su función redistribuidora de rentas. En el caso de la PAC, la redistribución es fundamentalmente sectorial al suponer sus pagos una transferencia real de riqueza desde la industria y los servicios a la agricultura, aunque también cabe hablar de una redistribución territorial: sus ayudas favorecen el crecimiento de las zonas atrasadas por ser la agricultura el sector dominante en ellas; además, su apartado política rural se ocupa de impulsar la cohesión del medio rural. La redistribución de la política de cohesión es principalmente territorial: destina la mayor parte de sus créditos a los Estados y regiones rezagados con el fin de acercar su renta a la media comunitaria. No faltan, pues, razones para calificar la PAC y la política de cohesión como políticas armonizadoras por cuanto la mayor parte de sus recursos se dirigen a actividades o áreas con menor renta.

La reforma a que están siendo sometidas tales políticas en la segunda mitad del ciclo 2007-2013 es otro elemento que expresa su importancia. Esta revisión obedece tanto a las limitaciones que han venido explicitando como a la necesidad de adecuarlas a la Estrategia Europa 2020; ${ }^{2}$ resulta inconcebible progresar

1 Es un Tratado suscrito por los doce países que componen la Comunidad Europea en ese momento. Su objetivo central es crear las condiciones para alcanzar el mercado interior con el fin de garantizar la libre circulación de bienes, servicios, capitales y personas. Este objetivo se hace realidad el $1^{\circ}$ de enero de 1993. Es el primer Tratado en el que se dedica un capítulo a la cohesión económica y social.

2 Es la estrategia de crecimiento de la uE para la próxima década. Adoptada en el Consejo Europeo de 2010, establece tres objetivos: crecimiento inteligente, crecimiento sostenible y crecimiento integrador. 


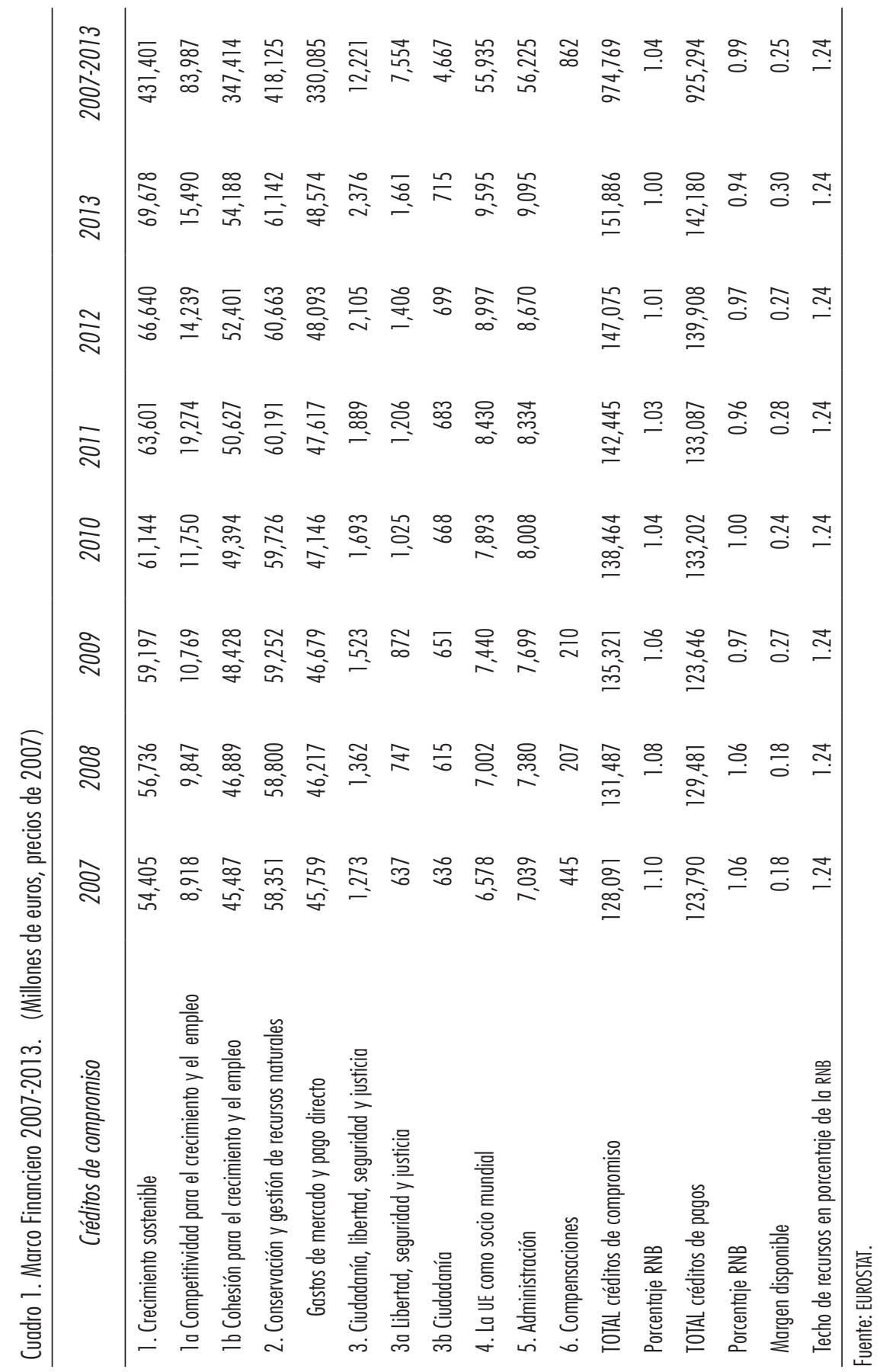


en una Europa más cohesionada sectorial y territorialmente, como reza dicha Estrategia, sin la aportación de estas políticas.

El hecho de que ambas políticas compongan la mayor parte del Presupuesto de la UE, tengan una dimensión eminentemente armonizadora, estén siendo sometidas a revisión para adecuarlas a la Estrategia Europa 2020 y ésta les conceda una particular importancia en la consecución de los objetivos propuestos para la próxima década, son, a nuestro entender, motivos suficientes para acometer su estudio en la recta final del ciclo plurianual 2007-2013.

\section{EL DEVENIR DE LA POLÍTICA DE COHESIÓN}

Una de las primeras cuestiones a considerar de la política de cohesión es que, pese a tener que esperar al Acta Única para institucionalizar el concepto de "cohesión económica y social", la consecución de este objetivo ha sido, en mayor o menor medida, una constante de la política europea desde el Tratado de Roma de 1957. Es cierto que tal noción no aparece en este Tratado, pero también lo es que su Artículo 2 asigna a la Comunidad la misión de impulsar un desarrollo armónico de las actividades económicas, crecimiento continuo y equilibrado, estabilidad creciente y elevación acelerada del nivel de vida de sus ciudadanos. Aun con todo, será a partir de 1986 cuando formalmente se fijen sus objetivos, no sus contenidos específicos, algo está todavía por definir, de ahí que la citada noción continúe sin liberarse de cierta carga de ambigüedad.

El Artículo 130 A del Acta Única establece que "a fin de promover un desarrollo armonioso del conjunto de la Comunidad, ésta desarrollará y proseguirá su acción encaminada a reforzar su cohesión económica y social. La Comunidad se propondrá, en particular, reducir las diferencias entre las diversas regiones y el retraso de las menos favorecidas". Por su parte, el Artículo 130 B completa este mandato con la obligación, tanto de los Estados miembros como de la Comunidad, de diseñar políticas que contribuyan a reforzar la cohesión económica y social. En este mismo artículo se enuncia que "la Comunidad apoyará dicha consecución con la acción que lleva a cabo por medio de los fondos con finalidad estructural". Finalmente, el Artículo 130 D encarga a la Comisión la presentación de una propuesta global de reforma de funcionamiento de los Fondos Estructurales (FFEE, a partir de ahora), tras los acuerdos adoptados en el Consejo de 24 de junio de 1988; esta reforma entra en vigor en 1989. 
El Tratado de Maastricht o de la Unión Europea (TUE) de 1992, en su Artículo B, enuncia que entre los objetivos de la Unión se halla el "fortalecimiento de la cohesión económica y social”. En su versión consolidada, el Artículo 2 reza que uno de los objetivos de la Unión Europea es "promover el progreso económico y social y un alto nivel de empleo y conseguir un desarrollo equilibrado y sostenible, principalmente mediante la creación de un espacio sin fronteras interiores, el fortalecimiento de la cohesión económica y social...”. Debe subrayarse también que en su Protocolo sobre la cohesión económica y social, las Altas Partes Contratantes "reafirman que el fomento de la cohesión económica y social es vital para el pleno desarrollo y éxito continuado de la Comunidad y destacan la importancia de que en los Artículos $2^{\circ}$ y $3^{\circ}$ del Tratado se incluya la cohesión económica y social".

Con el objeto de alcanzar una mayor cohesión económica y social, se decide crear el Fondo de Cohesión (FC, a partir de ahora), ésto va a aportar recursos a los países cuya renta per cápita no alcance el $90 \%$ de la media comunitaria en PPS (paridad de poder adquisitivo), y ampliar considerablemente los créditos destinados a los FFEE (FEDER y FSE). La creación del FC, según el Reglamento (CE) no 1164/1994, culmina institucionalmente la política emprendida con el Acta Única y fortalece el principio que subyace tras el objetivo de cohesión económica y social: el de solidaridad. La cohesión económica y social se convierte a partir de este momento en una de las políticas vertebradoras de la UE, al considerarse que contribuirá a reducir la inquietante dispersión de renta de países y regiones e impulsar el crecimiento económico común, eso sí, en plano subsidiario respecto de las políticas económicas nacionales.

Igual que el TUE, el Tratado Constitutivo de las Comunidades Europeas (TCE), en su versión consolidada, consagra la cohesión económica y social como uno de los objetivos y misiones de la UE. En su Artículo 2 (antes también Artículo 2) se recoge la función de promover: un desarrollo armónico, equilibrado y sostenible; alto grado de competitividad y convergencia de los resultados económicos; elevación del nivel de vida; cohesión económica y social; y solidaridad entre los Estados miembros. Su Artículo 3 (antes también Artículo 3) indica que para alcanzar los fines enunciados en el Artículo 2, la acción de la Comunidad impulsará "el fortalecimiento de la cohesión económica y social". En el Artículo 158 (antes 130 A) se expresa que "a fin de promover un desarrollo armonioso del conjunto de la Comunidad, ésta desarrollará y proseguirá su acción encaminada a reforzar su cohesión económica y social. La Comunidad se propondrá, en particular, reducir las diferencias entre los niveles de desarrollo de las diversas regiones y el retraso de las regiones o islas menos favorecidas, incluidas las zonas rurales". En el 
Artículo 159 (antes 130 B) se establece que "los Estados miembros conducirán su política económica y la coordinación con miras a alcanzar también los objetivos enunciados en el Artículo 158. Al formular y desarrollar las políticas y acciones de la Comunidad y desarrollar el mercado interior, se tendrán en cuenta los objetivos enunciados en el Artículo 158, participando en su consecución. La Comunidad apoyará asimismo dicha consecución a través de la actuación que realiza mediante los fondos con finalidad estructural (Fondo Europeo de Orientación y de Garantía Agrícola, sección Orientación; Fondo Social Europeo; Fondo Europeo de Desarrollo Regional), el Banco Europeo de Inversiones y los otros instrumentos financieros existentes". Por último, el Artículo 160 (antes 130 C) fija que "el Fondo Europeo de Desarrollo Regional estará destinado a contribuir a la corrección de los principales desequilibrios regionales dentro de la Comunidad mediante una participación en el desarrollo y en el ajuste estructural de las regiones menos desarrolladas y en la reconversión de las regiones industriales en declive".

Los Tratados de Ámsterdam y Niza no introducen novedades dignas de mención, sí lo hace el Tratado de Lisboa. En primer término, incorpora la cohesión territorial a la cohesión económica y social de los Tratados anteriores, dando lugar a la primera modificación de cierta importancia de la política de cohesión, de modo que a partir de entonces hay que hablar de la dimensión económica, social y territorial de la política de cohesión; prueba de esta inquietud por la cohesión territorial es la adopción del Libro Verde en 2008. ${ }^{3}$ Por otra parte, refuerza la idea de que la política de cohesión económica, social y territorial sea algo más que un mecanismo de solidaridad, que era la idea que subyacía desde el Acta Única. Partiendo de esta consideración, la política de cohesión queda directamente implicada en aumentar la competitividad de la UE en los mercados internacionales.

La Estrategia Europa 2020, en su objetivo de conciliar la lucha contra la crisis económica, aumentar la competitividad, hacer frente a los nuevos retos y reducir las disparidades internas de la UE, plantea la necesidad de acometer una reforma ambiciosa de la política de cohesión para que ésta se implique en alcanzar dichas metas. Así puede entenderse que la inserte en el objetivo crecimiento económico, que aparece como eje central y vertebrador de la política comunitaria. La meta prioritaria seguirá siendo reducir la dispersión

3 El Libro Verde abre un debate sobre la política de cohesión territorial con el objeto de impulsar la competitividad y el desarrollo sostenible. 
territorial de la renta y un crecimiento más armónico, pero se introducen una serie de novedades, como son: extender la política de cohesión a toda la UE; impulsar la investigación, la innovación y el desarrollo del capital humano con el fin de incrementar la productividad y la competitividad; por último, intensificar la coordinación entre la política de cohesión y el resto de las políticas con objeto de ampliar su eficacia. Detrás de estas novedades subyace una que engloba todas ellas: es conveniente restar cierto protagonismo a la ayuda a países y regiones más atrasadas y prestar mayor atención al tejido productivo de las zonas más prósperas y competitivas con el objeto de que generen un efecto arrastre sobre las demás.

De lo expuesto sobre el devenir de la política de cohesión cabe concluir que estamos ante una política cuyo objetivo ha ido experimentando diversos cambios a medida que han ido apareciendo nuevos retos para la UE. Por tanto, sus modificaciones explicitan los desafíos con los que se ha ido enfrentado la UE durante estas décadas. Así, cabe explicar que, ante el debilitamiento europeo en los mercados internacionales, se haya decidido: implicar más directamente a la política de cohesión en el objetivo competitividad; disminuir sus prioridades para evitar que siguiera siendo una especie de cajón de sastre donde cabe meter un sinfín de actuaciones; perseguir una mayor eficiencia para hacer más rentables sus cuantiosas inversiones; finalmente, fortalecer la cohesión territorial. A pesar de todo, el objetivo central de la política de cohesión sigue siendo impulsar el crecimiento de los países y las regiones más atrasadas.

\section{EL DEBILITAMIENTO DE LA POLÍTICA DE CONVERGENCIA}

En el Marco Financiero 2007-2013, los créditos destinados a la política de cohesión están agrupados en la subrúbrica 1b, Cohesión para el crecimiento y el empleo (Cuadro 1). Se elevan a 347,407 millones de euros a precios corrientes y representan el $35.6 \%$ del Presupuesto, lo que revela la importancia que la UE27 concede a dicha política. Pero quizás lo más significativo sea que, mientras el gasto total aumenta el $29.6 \%$ en relación con las Perspectivas Financieras 2000-2006, el de cohesión o acciones estructurales lo hace el 33.1\%, ${ }^{4}$

4 Al objeto de unificar criterios para las etapas 2000-2006 y 2007-2013, entendemos por recursos para la cohesión en ambos ciclos los correspondientes a los FFEE y FC. 
convirtiéndose en la subrúbrica más voluminosa, seguida de los gastos de mercado y pagos directos a la agricultura (330,085 millones de euros). A tenor de estos datos, todo parece indicar, en principio, que en el ciclo 2007-2013 asistimos a un reforzamiento de la política de cohesión en comparación con el periodo 2000-2006.

Lo acontecido en los presupuestos anuales para los ejercicios 2007 a 2010 indica que los recursos destinados a acciones estructurales evolucionan de un modo dispar a lo establecido en el Marco Financiero: frente a un incremento del $11.9 \%$ del gasto total, los créditos para la cohesión lo hacen el 8.6\%, lo que sienta la bases para que su presencia porcentual descienda del $36 \%$ en 2007 al 34.9 en 2010. En la primera mitad del Marco Financiero 2007-2013 los créditos para la cohesión experimentan un significativo aumento en términos absolutos, pero una caída porcentual también significativa (Cuadro 2).

A tenor de los instrumentos financieros de la política de cohesión en el Marco Financiero 2007-2013, ${ }^{5}$ la estructura presupuestaria de esta política se caracteriza por el predominio de los FFEE, algo que ha venido sucediendo en todos los ciclos precedentes. La novedad que ofrecen los presupuestos anuales para los ejercicios 2007 a 2010 es una ralentización del crecimiento de los FFEE $(2.2 \%)$, frente a una ingente expansión del FC (43\%). Este crecimiento, muy superior al programado en el Marco Financiero 2007-2013, se explica por la voluntad comunitaria de atender las necesidades nacionales de los doce socios incorporados en 2004 y 2007, que tienen todos ellos acceso al FC por no alcanzar su renta per cápita el $90 \%$ de la media europea. ${ }^{6} \mathrm{~A}$ pesar de ello, en 2010 los FFEE representan el 79.3\% de los recursos destinados a la política de cohesión.

El cuantioso esfuerzo financiero que representa la expansión del FC queda confirmado al vincular sus créditos con el indicador básico empleado por la UE27 para distribuir los recursos para acciones estructurales: población be-

5 En el Marco Financiero 2007-2013, la política de cohesión persigue tres objetivos: Convergencia, Competitividad regional y empleo y Cooperación territorial europea. Los instrumentos financieros en esta política son los siguientes: en el Objetivo Convergencia, FEDER, FSE y FC; en el Objetivo Competitividad regional y empleo, FEDER y FSE; y en el Objetivo Cooperación territorial, FEDER.

6 En el ciclo plurianual 2007-2013 son considerados países-cohesión los Estados miembros cuya renta per cápita en PPS no alcanza el 90\% de la media comunitaria en el periodo 20012003. Estos países son: Grecia, Portugal y los doce incorporados en 2004 y 2007. A estas catorce economías se añade España, que se halla en régimen de phasing-out. 
neficiaria. A precios constantes de 2004, los recursos del FC por población beneficiaria ascienden a 43.2 euros per cápita en la UE15 de 2003 (último ejercicio de la UE15), mientras se elevan a 67.6 euros en la UE27 de 2010, si se excluye España (en régimen de phasing out), y a 50.9 euros, si se la incluye. Hay que enfatizar que el reforzamiento del FC en la primera mitad del Marco Financiero 2007-2013 en relación con la UE15 se acompaña de una nota de particular interés: no siempre existe correspondencia entre recursos del FC por población beneficiaria y nivel de renta de los países receptores, lo que no es la mejor opción para reducir las disparidades territoriales.

Cuadro 2. Créditos de compromiso para políticas de cohesión, 2007-2010

Millones de euros. Precios corrientes

\begin{tabular}{|c|c|c|c|c|}
\hline & 2007 & 2008 & 2009 & 2010 \\
\hline Fondos Estructurales & $38,360.4$ & $39,100.9$ & $39,117.2$ & $39,191.8$ \\
\hline - Objetivo Convergencia & $28,079.9$ & $29,208.3$ & $29,723.2$ & $30,256.0$ \\
\hline - Empleo y asuntos sociales & $7,403.5$ & $7,614.8$ & $7,305.9$ & $7,473.7$ \\
\hline - Polífica regional & $20,676.5$ & $21,593.5$ & $22,417.3$ & $22,782.3$ \\
\hline - Objetivo Competitividad regional y empleo & $9,051.8$ & $8,592.5$ & $8,110.8$ & $7,604.8$ \\
\hline - Empleo y asuntos sociales & $3,670.0$ & $3,483.8$ & $3,477.2$ & $3,343.8$ \\
\hline - Política regional & $5,581.8$ & $5,108.7$ & $4,633.5$ & $4,261.0$ \\
\hline - Objetivo Cooperación territorial & $1,133.1$ & $1,214.0$ & $1,198.7$ & $1,242.9$ \\
\hline - Asistencia técnica & 95.6 & 86.2 & 84.6 & 88.1 \\
\hline Fondo de Cohesión-Política regional & $7,126.4$ & $8,155.0$ & $9,296.6$ & $10,192.2$ \\
\hline $\begin{array}{l}\text { Total en cohesión para el crecimiento y } \\
\text { el empleo }\end{array}$ & $45,486.8$ & $47,255.9$ & $48,426.9$ & $49,387.6$ \\
\hline Total presupuesto & $126,383.2$ & $130,569.6$ & $136,576.1$ & $141,452.8$ \\
\hline
\end{tabular}

Fuente: Comisión Europea, Presupuestos Generales. 
Otro rasgo en la estructura presupuestaria de la política de cohesión en la primera mitad del Marco Financiero 2007-2013 es que la mayor parte de los FFEE tiene como destino el Objetivo Convergencia. Su continuo crecimiento, tanto en el Marco Financiero 2007-2013 como en los presupuestos anuales para los ejercicios 2007 a 2010, conduce a que tal Objetivo represente en este último ejercicio el $77.2 \%$ del total de FFEE. Ello no evita que asistamos a un hecho de especial calado para el Objetivo Convergencia territorial de la política de cohesión: si medimos esos recursos en términos de población beneficiaria, en la UE27 se produce una contracción respecto de la UE15.

Comparando los FFEE por población beneficiaria en las regiones convergentes de la UE15 en 2003 y de la UE27 en 2009, ${ }^{7}$ a precios constantes de 2004, observamos tal disminución (Cuadro 3). En 2003, los FFEE por población beneficiaria se elevan a 197.1 euros per cápita en tales regiones, mientras que en 2009 son 157.3 euros. La inquietud que despierta esta reducción se amplía al considerar dos hechos. Uno es que buena parte de la población beneficiaria en 2009 tiene una renta per cápita muy inferior a la del 2003 al pertenecer ésa, en gran medida, a socios incorporados en 2004 y 2007. El segundo hecho no es menos preocupante: en 2009, los FFEE por población beneficiaria ascienden a 194.4 euros per cápita en las regiones de la antigua UE15 y a 143.0 euros en las regiones de los doce socios nuevos, pese a disponer de una renta per cápita sensiblemente inferior que las primeras. Tal como hemos apuntado en otras oportunidades (González Temprano, 2012), los FFEe por población beneficiaria en la UE27 no siempre se corresponden con el nivel de renta de las regiones receptoras, algo que no puede dejar de sorprender si consideramos que una de las metas prioritarias de estos créditos es una Europa regionalmente más armónica.

Como se recoge en el denominado Informe Sapir, es más que polémico que regiones convergentes de países muy desarrollados, como las de Alemania, Francia, Italia y Reino Unido, se beneficien de estos FFEE, pero resulta más polémico aún que regiones de países ricos perciban recursos superiores a los de regiones con menor renta de países atrasados. Es cierto que algunos Estados miembros, particularmente los rezagados, tienen algunas limitaciones para cofinanciar proyectos o absorber grandes volúmenes de fondos, pero ello difícilmente justifica la distribución de los recursos para la convergencia del Marco Financiero 2007-2013.

7 En el Marco Financiero 2007-2013 se entiende por regiones convergentes aquellas cuya renta per cápita en PPs no alcanza el 75\% de la media comunitaria en la etapa 2000-2002. 
Cuadro 3. Fondos Estructurales para la convergencia regional, población beneficiaria y créditos per cápita, 2007-2009

\begin{tabular}{|c|c|c|c|}
\hline & 2007 & 2008 & 2009 \\
\hline \multicolumn{4}{|c|}{ Fondos Estructurales } \\
\hline \multicolumn{4}{|c|}{ Millones de euros. Precios 2004} \\
\hline Regiones de la UE15 & 10,978 & 10,978 & 10,978 \\
\hline Regiones de los doce socios nuevos & 13,112 & 13,530 & 13,964 \\
\hline Total regiones convergentes UE27 & 24,090 & 24,508 & 24,942 \\
\hline Regiones UE27 phasing out & 2,369 & 2,174 & 1,979 \\
\hline TOTAL & 26,460 & 26,683 & 26,921 \\
\hline \multicolumn{4}{|c|}{ Población beneficiaria } \\
\hline Regiones de la UE15 & 56,130 & 56,317 & 56,480 \\
\hline Regiones de los doce socios nuevos & 97,877 & 97,739 & 97,623 \\
\hline Total regiones convergentes UE27 & 154,007 & 154,056 & 154,103 \\
\hline Regiones UE27 phasing out & 16,911 & 16,987 & 17,055 \\
\hline TOTAL & 170,918 & 171,043 & 171,158 \\
\hline \multicolumn{4}{|c|}{ Fondos Estructurales per cápita } \\
\hline Regiones de la UE15 & 195.6 & 194.9 & 194.4 \\
\hline Regiones de los doce socios nuevos & 134.0 & 138.4 & 143.0 \\
\hline Regiones convergentes UE27 & 182.7 & 179.4 & 176.3 \\
\hline TOTAL & 154.8 & 156.0 & 157.3 \\
\hline
\end{tabular}

Fuente: González Temprano (2012).

De acuerdo con lo expresado sobre la política de cohesión, puede afirmarse, en la primera mitad del Marco Financiero 2007-2013 se refuerza el papel armonizador del FC, al tiempo que se reduce el de los FFEE en comparación con la etapa de la UE15. Debido a que la mayor parte de los recursos destinados a la política de cohesión son FFEe, se colige que durante estos años se produce globalmente un debilitamiento de dicha política; la contracción relativa de los FFEE no es universal: las regiones afectadas pertenecen a los países incorporados en 2004 y 2007, mientras las pertenecientes a la antigua UE15 mantienen valores del ciclo anterior. 
En consecuencia, la decisión de la UE15 de abrir sus puertas hacia el Este de Europa no se ha acompañado del esfuerzo financiero que hubiera garantizado a las nuevas regiones convergentes dispusieran, al menos, de los mismos recursos que las ubicadas en la UE15. Ello conduce a que los pagos que reciben los socios de la UE27 para políticas de cohesión dibujen dos escenarios diferenciados: uno, constituido por las doce economías avanzadas, el otro, integrado por las catorce atrasadas o países-cohesión y, en medio de ambos, España, que se encuentra en una situación de transición tanto en la percepción de pagos de la UE como en su relación con el FC (Cuadro 4).

En los doce países más prósperos, los pagos para políticas de cohesión son minoritarios tanto en el seno de lo que hemos denominado políticas armonizadoras como en el total de recursos que reciben de la UE. Ello sucede aun en Alemania, pese a ser uno de los tres grandes receptores de estos pagos (los otros dos son Polonia y Espańa) y tener regiones convergentes. Estos pagos representan una cantidad anecdótica en porcentaje del PIB, de manera que contribuyen escasamente al crecimiento económico de tales países. En 2009, este porcentaje no sobrepasa el $0.1 \%$, salvo en Italia, que alcanza el $0.2 \%$; en España, se eleva al $0.4 \%$.

En los catorce países rezagados, los pagos para políticas de cohesión conforman la mayor parte de las políticas armonizadoras, así como de los recursos totales que perciben de la UE, pese a que su sector agrario tiene una relevancia bastante superior a la que presenta en las más ricas. Por tanto, las políticas armonizadoras tienen en estos catorce países un carácter más territorial que sectorial, lo contrario de lo que acontece en las economías desarrolladas.

Según esta nota y la vocación redistributiva de la política de cohesión, los pagos de ésta se hallan concentrados en las catorce economías rezagadas o países-cohesión: en 2009, son el destino del 57\% de dichos recursos, cuando su población representa el $25.1 \%$ de la UE27. El rasgo redistributivo de dichos pagos se observa con particular nitidez en los países cuyo territorio es convergente en su totalidad: Bulgaria, Estonia, Letonia, Lituania, Malta, Polonia, Rumanía y Eslovaquia. Exceptuando Bulgaria y Rumanía por no haber entrado en funcionamiento todos los mecanismos de integración, los cinco países restantes son en 2009 el destino del $26.1 \%$ de pagos para políticas de cohesión, mientras que su población sólo representa el 10.2\% de la UE27; la otra cara de la moneda son España, Grecia y Portugal: en esa misma fecha reciben el $26.1 \%$ de los citados pagos y su población significa el $13.6 \%$ de la UE27, pero con una renta per cápita superior a la de esas cinco economías. 
Cuadro 4. Pagos de la UE a los Estados miembros para acciones estructurales, 2007-2009. Millones de euros

\begin{tabular}{|c|c|c|c|c|c|c|}
\hline & \multicolumn{3}{|c|}{ Política de cohesión } & \multirow{2}{*}{$\begin{array}{c}\text { Polit. cohesión/ } \\
\text { Pagos totales } \\
\%\end{array}$} & \multirow{2}{*}{$\begin{array}{c}\text { Polít. } \\
\text { cohesión/Pol. } \\
\text { Armonizadoras } \\
\%\end{array}$} & \multirow{2}{*}{$\begin{array}{c}\text { Polít. cohesión/ } \\
\qquad P I B \\
\%\end{array}$} \\
\hline & & & & & & \\
\hline & 2007 & 2008 & 2009 & 2009 & 2009 & 2009 \\
\hline Bélgica & 346.9 & 397.4 & 370.8 & 15.0 & 26.8 & 0.1 \\
\hline Bulgaria & 144.9 & 223.0 & 267.0 & 27.6 & 42.4 & 0.8 \\
\hline República Checa & 918.3 & $1,679.4$ & $1,974.9$ & 66.1 & 68.0 & 1.4 \\
\hline Dinamarca & 129.3 & 98.0 & 56.5 & 4.0 & 4.5 & 0.0 \\
\hline Alemania & $4,311.8$ & $3,082.1$ & $3,311.4$ & 27.9 & 30.9 & 0.1 \\
\hline Estonia & 216.8 & 237.0 & 517.3 & 72.8 & 76.7 & 3.7 \\
\hline Irlanda & 263.7 & 213.6 & 121.3 & 6.3 & 6.7 & 0.1 \\
\hline Grecia & $4,592.6$ & $4,712.4$ & $2,259.2$ & 41.4 & 43.3 & 1.0 \\
\hline España & $5,429.6$ & $4,246.7$ & $4,174.2$ & 35.1 & 37.6 & 0.4 \\
\hline Francia & $2,449.6$ & $2,312.6$ & $1,920.4$ & 13.5 & 15.6 & 0.1 \\
\hline Italia & $4,452.6$ & $3,688.9$ & $2,413.3$ & 25.1 & 29.2 & 0.2 \\
\hline Chipre & 34.4 & 47.3 & 74.1 & 37.6 & 55.4 & 0.4 \\
\hline Letonia & 437.2 & 383.4 & 469.7 & 64.1 & 66.4 & 2.5 \\
\hline Lituania & 465.5 & 630.2 & $1,176.8$ & 61.8 & 70.6 & 4.3 \\
\hline Luxemburgo & 13.1 & 9.6 & 7.1 & 4.3 & 11.5 & 0.0 \\
\hline Hungría & $1,304.2$ & $1,188.6$ & $2,174.4$ & 57.6 & 59.0 & 2.2 \\
\hline Malta & 49.1 & 43.9 & 35.3 & 54.2 & 81.3 & 0.6 \\
\hline Países Bajos & 251.4 & 670.8 & 242.3 & 12.1 & 16.0 & 0.0 \\
\hline Austria & 264.7 & 231.9 & 241.2 & 13.2 & 15.2 & 0.1 \\
\hline Polonia & $4,217.1$ & $4,608.7$ & $6,082.0$ & 63.7 & 65.7 & 1.6 \\
\hline Portugal & $2,456.1$ & $2,568.9$ & $2,433.6$ & 65.3 & 68.5 & 1.5 \\
\hline Rumanía & 421.4 & 648.5 & 918.1 & 31.3 & 43.9 & 0.7 \\
\hline Eslovenia & 162.0 & 234.6 & 368.3 & 57.9 & 62.4 & 1.1 \\
\hline Eslovaquia & 635.2 & 809.5 & 584.8 & 47.3 & 50.7 & 0.9 \\
\hline Finlandia & 322.0 & 213.7 & 193.9 & 15.8 & 16.8 & 0.1 \\
\hline Suecia & 303.5 & 153.7 & 212.2 & 14.5 & 18.9 & 0.1 \\
\hline Reino Unido & $2,357.0$ & $2,100.3$ & $1,274.9$ & 20.5 & 24.7 & 0.1 \\
\hline Varios & 24.3 & 76.0 & 57.9 & & & \\
\hline Total & $36,974.0$ & $35,510.7$ & $33,932.9$ & 33.6 & 37.8 & 0.3 \\
\hline
\end{tabular}

Fuente: Elaboración propia con datos del Ministerio de Economía y Hacienda. 
La relevancia de los pagos para políticas de cohesión en porcentaje del PIB es mucho mayor en los países atrasados que en los ricos. En 2009, se sitúa entre el 1 y el 2\%, aunque hay miembros, como Estonia y Lituania, que superan el 3\%.

Sin obviar los interrogantes que pueden suscitar diversos apartados de la política de cohesión del Marco Financiero 2007-2013, como son la distribución de recursos, dispersión en un sinfín de objetivos, bajo nivel de eficiencia o anteposición de intereses nacionales y regionales a comunitarios, tal como se asume en la comunicación Revisión del Presupuesto de la UE [СОМ (2010) 700, de 19 de octubre de 2010], parece difícil refutar que esta política ha sido motor de cambio en los últimos diez ańos, particularmente en los países y regiones atrasadas.

\section{DIVERSIDAD Y RETOS DE LA AGRICULTURA EUROPEA}

En el Marco Financiero 2007-2013, los créditos destinados al mantenimiento de los recursos naturales componen la Rúbrica 2, denominada Conservación y gestión de los recursos naturales (Cuadro 1). Ascienden a 418,125 millones de euros, a precios corrientes, y representan el $42.9 \%$ del Presupuesto plurianual. Es la rúbrica más voluminosa.

En torno a esta rúbrica conviene tener presente tres consideraciones. $\mathrm{Su}$ crecimiento en el Marco Financiero 2007-2013 es cuantioso respecto de la etapa 2000-2006 (25.3\%), pero inferior al del Presupuesto total (29.6\%), por lo que en el ciclo actual queda mermada la presencia porcentual detentada en 2000-2006 (44.3\%). Su crecimiento entre estos dos ciclos es notablemente inferior al de la política de cohesión (33.1\%), en conclusión, el Marco Financiero 2007-2013 continúa aminorándose la diferencia entre ambas políticas. Por último, la política agraria, cuyos créditos representan la práctica totalidad de la política de recursos naturales, está inserta desde 2003 en un conjunto de reformas entre cuyos objetivos figura el control del gasto.

La política de contención del gasto en recursos naturales acordada para el Marco Financiero 2007-2013 es confirmada por los presupuestos anuales para los ejercicios 2007 a 2010. En estos cuatro presupuestos tales recursos aumentan el 6.5\%, mientras que el Presupuesto total lo hace el 11.9\% (Cuadro 5), pasando de significar el $44.2 \%$ en 2007 a ser el $42.1 \%$ en 2010; en este último ejercicio tales créditos representan ya un porcentaje inferior al decidido para todo el ciclo $(42.9 \%)$. 
Otro aspecto a tener presente de los créditos para conservación y gestión de los recursos naturales, tanto en el Marco Financiero 2007-2013 como en los presupuestos anuales para los ejercicios 2007-2010, es el dominio absoluto del sector agrario, como hemos adelantado. En estos cuatro presupuestos anuales la política agraria representa alrededor del $98 \%$ de la Rúbrica 2. Pasamos a analizar esta política.

Cuadro 5. Créditos de compromiso en conservación y gestión de los recursos naturales, 2007-2010. Millones de euros a precios corrientes

\begin{tabular}{lrrrr}
\hline & 2007 & 2008 & 2009 & 2010 \\
\hline Gastos de mercado y ayudas directas & 42,312 & 41,006 & 41,042 & 43,820 \\
$\quad$ Mercados agrícolas & 41,971 & 40,562 & 40,646 & 43,417 \\
Desarrollo rural & 12,371 & 13,303 & 14,002 & 14,364 \\
Fondo Europeo de la Pesca & 571 & 612 & 632 & 644 \\
Gobierno y otros & 596 & 639 & 656 & 671 \\
(Life y otros programas) & & & & \\
Total & 55,850 & 55,560 & 56,332 & 59,499 \\
\hline
\end{tabular}

Fuente: Comisión Europea, Presupuesto General de la Unión Europea. Resumen de datos.

Uno de los rasgos que ofrece la agricultura europea es la diversidad. Es cierto que ésta es un valor añadido y, como tal, fuente potencial de competitividad, pero también lo es de tal diversidad se derivan inquietantes desafíos, como son el minifundismo parcelario y fundiario, disparidad de modelos de producción y gestión, redes de distribución que encarecen sobremanera el precio final de los alimentos al generar ingentes brechas entre precios percibidos por los agricultores y abonan a los consumidores, por no hablar de rentas agrarias por UTA bastante inferiores al resto de las actividades económicas. En consecuencia, la PAC se enfrenta con el reto de tener que operar en una realidad particularmente compleja, reto que se ve acentuado con las ampliaciones de 2004 y 2007, de ahí que los valores medios de la UE27 se deban examinar con mayor cautela que los referidos a la UE15.

Las disparidades agrarias entre Estados miembros son de muy diversa índole. Una de ellas, quizás la más significativa, es el diferente peso de la producción y empleo agrarios en la economía nacional. Por ejemplo, los datos de Alemania, Luxemburgo, Eslovaquia, Suecia y Reino Unido poco tienen que 
ver con los de Bulgaria, Grecia, Lituania, Portugal y Rumanía. Estos dos grupos de países ejemplifican las amplísimas diferencias internas en producción y empleo agrarios y los nexos entre éstos y nivel de renta per cápita.

Otro parámetro donde pueden constatarse las disparidades nacionales es el VAB por hectárea cultivada. En 2009, los países de la UE15 arrojan una cuantía de 1,052 euros, mientras que en los doce socios incorporados en 2004 y 2007 es 367.7 euros (Cuadro 7); por otra parte, los datos de Bélgica, Grecia, Italia, Chipre, Malta y Países Bajos guardan escasa relación con los de Bulgaria, República Checa, Estonia, Irlanda, Letonia, Lituania, Hungría, Eslovaquia y Suecia. Algo similar puede afirmarse del número de utAs por explotación y de hectáreas cultivadas por UTA.

Pasamos a examinar la política que regula la complejidad del sector agrario, la PAC y las respuestas que da a los desafíos enunciados.

\section{EVOLUCIÓN RECIENTE Y PROSPECTIVA DE UNA NUEVA PAC}

Las transformaciones experimentadas por la PAC desde su puesta en funcionamiento en 1962 no han evitado que hayan permanecido inalterables tres objetivos: incrementar la productividad, garantizar el abastecimiento a precios razonables y asegurar cierto nivel de ingresos a su población activa. A estos objetivos se han ido incorporando los derivados de los cambios operados en el seno de la uE y en su entorno, como son preservar la calidad y seguridad de los alimentos, respetar el medioambiente y el bienestar de los animales, desarrollar las zonas rurales y fomentar la competitividad.

La configuración actual de la PAC se inicia en los años noventa. La reforma de 1992 supone la ruptura con una etapa en la que las medidas estaban centradas en actuar sobre la oferta, al basarse en la disminución de los precios institucionales de algunos productos básicos y compensar, mediante pagos directos, el impacto de dichos recortes sobre la renta del productor. En 1995, la Comisión presenta a los Estados miembros una nueva estrategia en la que destaca la necesidad de adaptarse a los cambios sucedidos en el entorno agrario y dar cobertura al desarrollo del medio rural; este giro es recogido en el primer Informe de Cohesión de 1996, que aboga por una reforma de la PAC fundamentada en un mayor acercamiento de los precios institucionales al mercado y un enfoque multifuncional de desarrollo rural. En 1997, la Comisión ofrece un proyecto sobre el futuro de la política de la Unión (Agenda 2000) que contiene un conjunto de modificaciones de la PAC; con él se pretende dar respuesta a los retos que plantea el nuevo milenio a la agricultura y al medio rural. Atendiendo 
a estas propuestas, el Consejo Europeo de Berlín de 1999 aprueba la reforma de la PAC con diez nuevos Reglamentos y los recursos para su financiación.

La reforma de 1999 se resume en los términos siguientes: introduce precios institucionales más reducidos con el fin de fomentar la competencia; incrementa las ayudas directas para compensar la disminución de los precios institucionales; amplía las normas tendentes a garantizar la calidad y seguridad de los alimentos y el bienestar de los animales; integra los objetivos medioambientales en la PAC; sienta los fundamentos para una nueva política de desarrollo rural y convierte ésta en el segundo pilar de la PAC; descentraliza la gestión; por último, simplifica la estructura de la PAC.

Los cambios enunciados culminan con las reformas que se extienden de 2003 a 2006. A tenor del Reglamento (CE) no 1782/2003, los Reglamentos del Consejo de 2004 y 2006 y el Reglamento de la Comisión de 2005, se introduce un conjunto de novedades que modifica notablemente los mecanismos vigentes. Los ejes fundamentales del nuevo modelo son el desacoplamiento de las ayudas directas (Régimen de Pago Único), la modulación y la condicionalidad. Es un modelo de ayudas que se alínea con la apertura comercial para facilitar acuerdos con la OMC.

El principio de desacoplamiento de las ayudas directas o Régimen de Pago Único establece un sistema de pagos directos al productor disociados de la producción. El principio de modulación y disciplina financiera, por un lado, implanta de modo obligatorio y para toda la UE un mecanismo de reducción de los pagos destinados a las grandes explotaciones entre 2005 y 2012, por el otro, atiende las necesidades de las reformas previstas con el objeto de que los pagos de la PAC se mantengan en los límites fijados en el Consejo de Bruselas de 2002. El principio de condicionalidad implica la supeditación de la concesión de ayudas directas al cumplimiento de las normas comunitarias en materia de salud pública, medioambiente y salud y bienestar de los animales.

El acuerdo sobre el denominado chequeo médico de la PAC, decidido en el Consejo Europeo de Ministros de 2008, y la comunicación Una PAC simplificada para Europa: un beneficio para todos, presentada por la Comisión en marzo de 2009, cierran la ola de reformas iniciada en 1999 y abren una etapa de modificaciones que se aplicarán en el ciclo plurianual 2014-2020.

A las reformas acometidas en la PAC entre 1999 y 2009 conviene añadir dos acuerdos que afectan también a esta política: la reforma de sus instrumentos financieros y la Directriz agraria.

En aplicación del Reglamento (CE) no 1290/2005, se crea el Fondo Europeo Agrícola de Garantía (fEAGA) y el Fondo Europeo Agrícola de Desarrollo Rural (FEADER), que sustituyen al Fondo Europeo de Orientación y Garantía 
Agrícola. Los nuevos Fondos tienen, prácticamente, los mismos objetivos y financian las mismas medidas que las anteriores secciones del FEOGA.

La Linea directriz agraria o Directriz agraria, establecida por el acuerdo de Bruselas de 2002, impone el principio de estabilidad financiera para el conjunto del gasto agrario. Según este principio, en el Marco Financiero 2007-2013 el gasto no puede superar el umbral de la nueva Directriz fijada en Berlín para el ejercicio 2006, incrementada en 1\% anual como compensación a la inflación. La realidad ha mostrado que los techos de gasto establecidos, más que limitadores, se han convertido de facto en garantía de gasto, lo que ha vaciado de contenido la Directriz. No puede sorprender que ésta haya acabado por desaparecer del Marco Financiero 2007-2013.

Las reformas de finales del siglo pasado y principios del actual no han impedido que la PAC se enfrente a retos que fuerzan a dar por concluido el régimen actual en 2013 e iniciar una nueva etapa en el ciclo plurianual 20142020. La segunda mitad del Marco Financiero 2007-2013 es, pues, testigo de propuestas de reformas que han de estar aprobadas antes de que finalice 2013. La PAC, igual que la política de cohesión, se desenvuelve durante estos años en un clima de transitoriedad.

Las causas que justifican una nueva reforma de la PAC responden, según la Comisión, a la necesidad de afrontar tres desafíos. Uno es la seguridad alimentaria: la PAC ha de afrontar la creciente demanda de alimentos y volatilidad de precios en los mercados internacionales, con las correspondientes consecuencias sobre la renta agraria, ${ }^{8}$ y la exigencia social de ampliar las normas de calidad y seguridad alimentaria. Un segundo reto es el medioambiente y cambio climático: si bien los gases de efecto invernadero procedentes de la agricultura han sido reducidos en más de un 20\% desde 1990 y la ecocondicionalidad vincula los pagos únicos al cumplimiento de determinados estándares medioambientales, las perspectivas del cambio climático plantean nuevos desafíos en las diferentes zonas europeas. El tercer reto es el equilibrio territorial: la ampliación a veintisiete miembros ha incrementado la diversidad de las zonas rurales y explotaciones agrarias. Hacer frente a estos tres desafíos significa adaptar la PAC a los objetivos de la Estrategia Europa 2020.

Atendiendo a estos retos, el Comisario Europeo de Agricultura y Desarrollo Rural lanza en abril de 2010 un debate público sobre el futuro de la PAC. Sus

8 Mientras los precios agrícolas aumentan en los últimos años un 50\%, los precios de la energía lo hacen un 200\% y los de los fertilizantes un 150\%. En 2009, la renta agrícola europea cae sustancialmente, de forma que llega a representar el $40 \%$ del resto de la economía. En este mismo ańo, la renta por habitante en las zonas rurales es el $50 \%$ de las zonas urbanas. 
resultados son ofrecidos en la Conferencia La PAC post 2013, organizada en Bruselas en julio de ese año. Basándose en ésta y en los debates y/o dictámenes del Consejo, Parlamento Europeo, Comité Económico y Social Europeo y Comité de las Regiones, la Comisión redacta la comunicación [Сом (2010) 672], La PAC en el horizonte de 2020: Responder a los retos futuros en el ámbito territorial, de los recursos naturales y alimentarios. El 12 de octubre de 2011 la Comisión presenta el proyecto de reforma que se ha de aplicar en el ciclo 2014-2020. ${ }^{9}$

Este proyecto significa una transformación de la PAC mucho más aguda a la década precedente, particularmente en el ámbito de los pagos y el respeto al medioambiente. Sigue resultando polémico que, mientras se mantiene un sistema de pagos no siempre se incentiva la modernización agraria, no se dedique mayor esfuerzo a resolver dos grandes retos de la agricultura europea: excesiva fragmentación de la propiedad de la tierra y canales de comercialización que generan una distancia excesiva entre los precios en destino y los percibidos por los productores, a resultas de lo cual el ciudadano europeo ha de pagar impuestos para financiar las ayudas agrarias y abonar precios muy distanciados de los ingresos percibidos por los agricultores.

\section{EL GASTO DE LA PAC COMO INSTRUMENTO ARMONIZADOR}

En el Marco Financiero 2007-2013, la PAC se asienta en dos Pilares. El Pilar I se ocupa de proporcionar ayuda directa a los agricultores y apoyar las medidas de mercado; ambas funciones son financiadas en su totalidad con fondos comunitarios. El Pilar II tiene por objeto el desarrollo rural, dedicándose a producir bienes públicos medioambientales, impulsar la competitividad de los sectores agrícola y silvícola, diversificar la actividad económica y fomentar la calidad de vida del medio rural; a tenor del Reglamento de Desarrollo Rural 1628/2005, las medidas de desarrollo rural son cofinanciadas, los Estados disponen de autonomía en su diseño y el instrumento de financiación es el FEADER.

9 Esta reforma se centra en diez puntos fundamentales: ayudas a la renta para dinamizar el crecimiento; instrumentos de gestión de crisis más dinámicos y mejor adaptados para hacer frente a los nuevos retos económicos; un pago ecológico para preservar la productividad a largo plazo y los ecosistemas; inversiones suplementarias en investigación e innovación; una cadena alimentaria y más competitiva; estímulo de medidas agroambientales; facilitar la instalación de jóvenes agricultores; estímulo de empleo rural y espíritu empresarial; zonas frágiles; y una PAC más simple y eficaz. 
Aun cuando la vocación armonizadora de la PAC es eminentemente sectorial, no debe obviarse el rol que desempeña en la cohesión territorial. En primer término, la PAC contribuye en su conjunto a la cohesión territorial desde el momento en que la producción agraria tiene lugar mayoritariamente en zonas de renta baja; tal función ha sido puesta de manifiesto por el Libro Verde sobre la cohesión territorial cuando al referirse al Pilar I manifiesta "el apoyo que presta a los agricultores también tiene un importante impacto territorial a través de las actividades y los ingresos que mantiene en las zonas rurales y mediante el fomento de gestión del suelo". En segundo lugar, este rol cohesionador se recoge en los Ejes II (programa agroambiental, red Natura $2000 \ldots$...), III y IV (diversificación de actividades) del Pilar II; el Eje I, modernización y competitividad, está enfocado a la actividad productiva.

$\mathrm{Al}$ representar el gasto de la PAC la práctica totalidad de la Rúbrica 2 del Marco Financiero 2007-2013 (Cuadro 1), gran parte de lo enunciado cuando analizamos esta Rúbrica es aplicable al presupuesto de la PAC. Por lo tanto, no es necesario reiterarlo, aunque sí hacer algunas puntualizaciones.

Debido a las reformas de finales de siglo pasado y principios del milenio actual, el gasto de la PAC se atempera en los presupuestos anuales para los ejercicios 2007 a 2010 (Cuadro 6). Su crecimiento durante estos ańos (6.6\%) es sensiblemente inferior tanto al del Presupuesto total (11.9\%) como a la política de cohesión (8.6\%). El resultado es una pérdida de presencia porcentual en el gasto comunitario total: si en 2007 representa el 43.4\%, en 2010 es el $41.3 \%$ (Cuadro 6). Pese a esta contracción, el peso de la PaC en el Presupuesto para 2010 sigue siendo significativamente superior al de la política de cohesión (34.9\%). Aún con todo, los 58,498.2 millones de euros que la Unión destina a la PAC en 2010 suponen un gasto por ciudadano comunitario de 116.7 euros.

La estructura del gasto de la PAC en los presupuestos citados sigue caracterizándose por la enorme hegemonía de los Gastos de mercado y ayudas directas. Hay una novedad: la decisión de contener las ayudas directas e impulsar el desarrollo rural conduce a que el Pilar I crezca un 3.4\% y el Pilar II lo haga un 16.1\%. Aun así, en 2010 el 74\% de los recursos de la PAC se destina a Gastos de mercado y ayudas directas y el $24.6 \%$ a Desarrollo rural. Lógicamente, las otras dos partidas, Salud de los animales y cuestiones fitosanitarias y Medioambiente, son anecdóticas.

Antes de adentrarnos en la distribución por países de los pagos directos de la PAC conviene recordar, por una parte, que las 178,443 miles de hectáreas cultivadas en 2009 se reparten de un modo muy desigual entre los veintisiete Estados miembros, lo que sienta los fundamentos para un reparto de pagos también muy dispar entre ellos (Cuadro 7). Por otra parte, la subvención co- 
munitaria por hectárea cultivada asciende a 313.1 euros en 2009, y a 4,673.6 euros por UTA en 2007.

Cuadro 6. Créditos de compromiso destinados a la PAC, 2007-2010.

Millones de euros a precios corrientes

\begin{tabular}{|c|c|c|c|c|}
\hline & 2007 & 2008 & 2009 & 2010 \\
\hline Gastos de mercado y ayudas directas & $41,971,361$ & $40,562,340$ & $40,646,181$ & $43,417,420$ \\
\hline $\begin{array}{l}\text { Salud de los animales y } \\
\text { cuestiones fitosanitarias }\end{array}$ & 309,800 & 411,650 & 374,015 & 375,385 \\
\hline Desarrollo rural & $12,373,442$ & $13,303,259$ & $14,003,278$ & $14,368,065$ \\
\hline $\begin{array}{l}\text { Medioambiente } \\
\text { (Life y otros programas) }\end{array}$ & 243,620 & 268,944 & 330,150 & 337,355 \\
\hline Total & $54,898,223$ & $54,546,193$ & $55,353,624$ & $58,498,225$ \\
\hline
\end{tabular}

Fuente: Comisión Europea, Presupuesto General de la Unión Europea. Resumen de datos.

Alemania, Grecia, España, Francia, Italia, Polonia y Reino Unido, acumulan el $67.2 \%$ de la tierra cultivada en 2009 , lo que explica, en gran medida, que sean el destino del $65.7 \%$ de los pagos de la PAC en este ejercicio. A la elevada concentración personal de los pagos (el 20\% de los propietarios, aproximadamente, recibe el $80 \%$ de los pagos al disponer del $80 \%$ de la tierra) se añade la nacional.

En 2009, Francia es el país con mayor extensión de tierra cultivada (16.5\% de la Ue27), como tal, es el principal receptor de pagos (18.6\%). Sin embargo, no es fácil mantener que sea la nación más beneficiada por la PAC, si por ello entendemos la cantidad de euros recibida por hectárea cultivada. Ateniéndonos a este cociente, el país más beneficiado es Malta con 810 euros, y de los siete grandes receptores, Grecia, que arroja 774.2 euros; Francia, con 353.7 euros, se halla muy alejada de ocupar las primeras posiciones.

Continuando con los siete grandes países receptores, Polonia y Reino Unido ofrecen los pagos por hectárea más reducidos. La diferencia entre ambos es que el Reino Unido recibe como compensación el denominado "cheque británico", ${ }^{10}$ acuerdo que habría que preguntarse si no cuestiona uno de

10 Es una reducción, negociada en 1984, que se aplica a la contribución del Reino Unido al Presupuesto de la uE con el fin de compensar el reducido beneficio que extrae este país de la PAC. 
los principios en que se asienta la PAC: la solidaridad financiera. Solamente ateniéndonos a los pagos por UTA, la agricultura francesa aparecería como la más beneficiada, seguida a muy escasa distancia por la británica, pero no parece ser éste el indicador más apropiado para hacer comparativas.

Salvando Bulgaria y Rumanía, los pagos a los países-cohesión presentan dos grupos diferenciados. Uno está integrado por Grecia, Chipre, Malta, Portugal, Eslovenia y Eslovaquia, con pagos por hectárea similares, cuando no superiores, a la media europea. El segundo grupo está constituido por la República Checa, Estonia, Letonia, Lituania, Hungría y Polonia, cuyos pagos son sensiblemente inferiores a la media comunitaria. Ello denota que no existe correspondencia entre pagos directos y renta per cápita, fenómeno que acontece también en las economías avanzadas. Si a este rasgo se añade que la mayor parte de la tierra cultivada de los socios incorporados en 2004 presenta pagos por hectárea inferiores a la media de la UE27, es fácilmente comprensible que la Comisión, en aras de la equidad y convergencia territorial, haya formulado una propuesta de reforma para el ciclo 2014-2020.

En resumen, los pagos directos de la PAC muestran acusadas diferencias nacionales tanto en el cociente por hectárea como por UTA. El hecho de que muchas de estas desigualdades sean difícilmente justificables y fuente de malestar, empuja a la Comisión, en su propuesta de Presupuesto para 20142020 [COM (2011) 500 final PARTE II], a reconocer inquietantes deficiencias en el sistema de pagos imperante en el Marco Financiero 2007-2013 y proponer su reforma, expresando que ésta "incluirá un sistema de convergencia para reducir estas disparidades y promover una distribución más justa de la ayuda financiera. Este reequilibrio de la ayuda es un elemento importante de la reforma encaminado a hacer un uso más eficaz de los recursos presupuestarios a través de pagos directos más equitativos y mejor dirigidos". ${ }^{11}$

11 En la misma Comunicación la Comisión indica que la distribución más justa y eficaz de las ayudas "se conseguirá de la siguiente manera: para todos los Estados miembros con pagos directos por debajo del $90 \%$ de la media de la uE27 se suprimirá, a lo largo del periodo, un tercio de la diferencia entre su nivel actual y el $90 \%$ de la media de los pagos directos de la UE. La asignación de ayuda para el desarrollo rural también se modernizará, con cuotas determinadas sobre la base de una serie de criterios económicos y territoriales objetivos que reflejen los futuros objetivos económicos, sociales, medioambientales y de política territorial". 


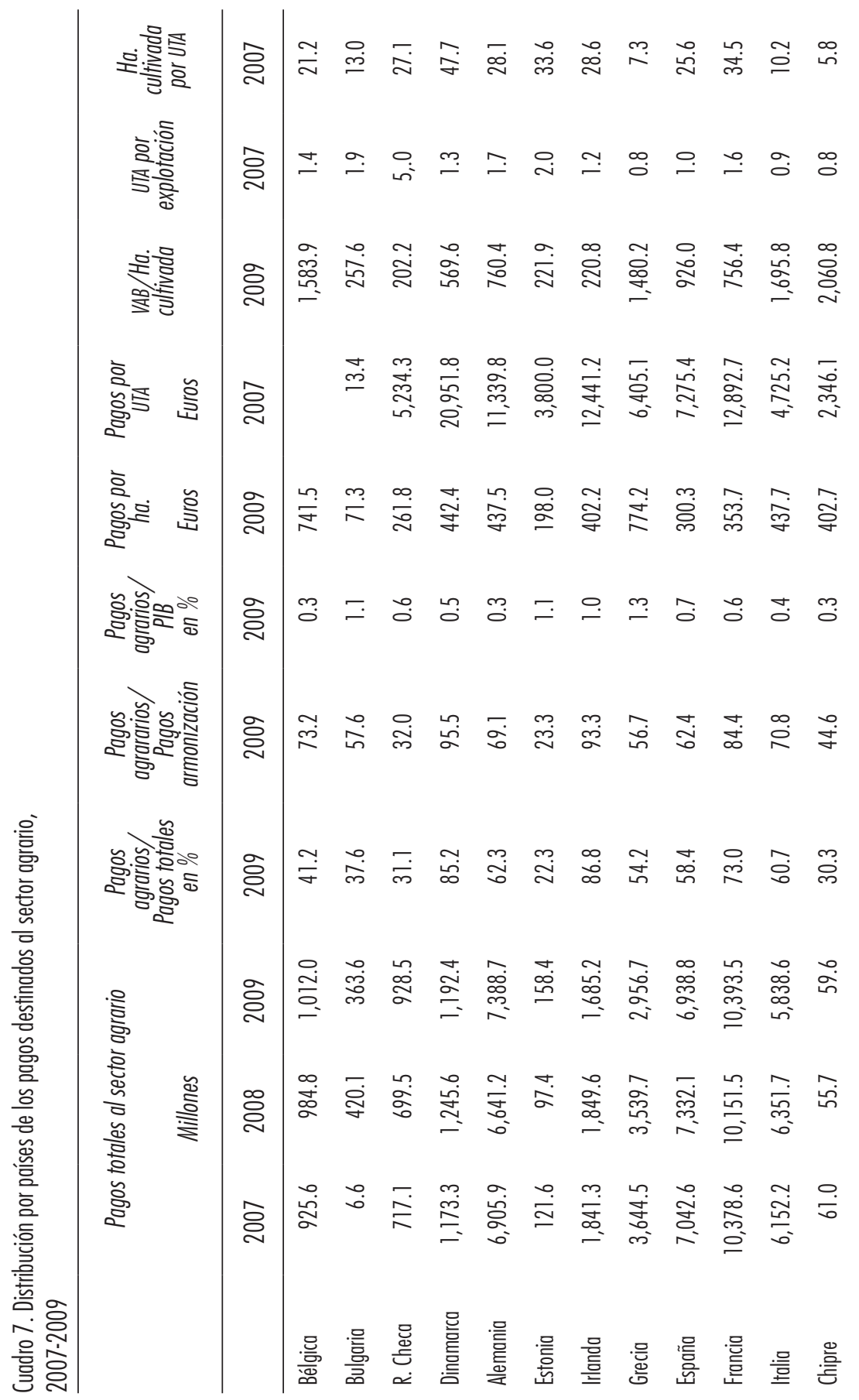




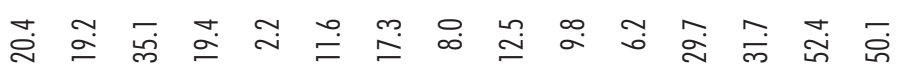

$\stackrel{a}{=}$

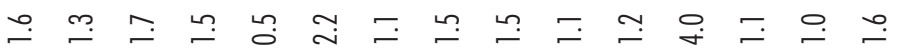

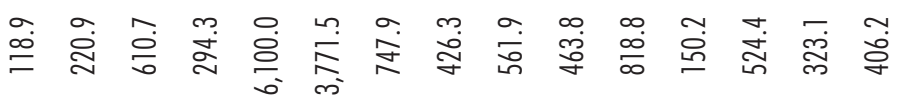

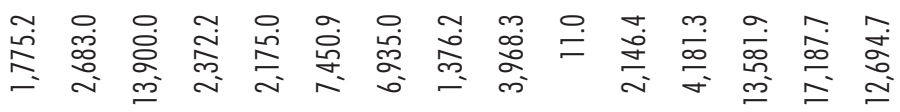

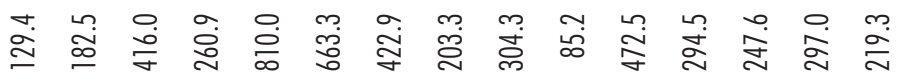

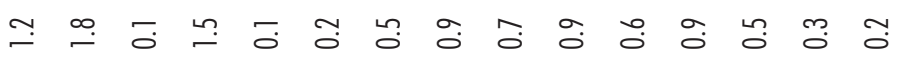

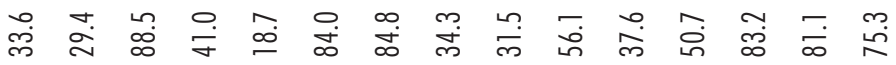

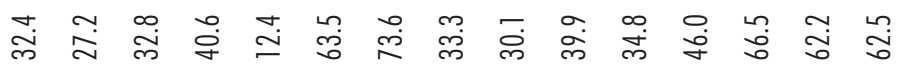

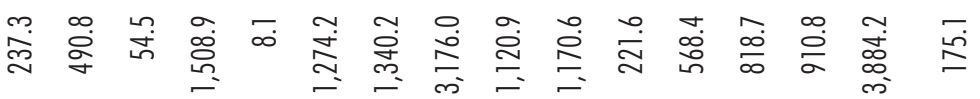

芯 总

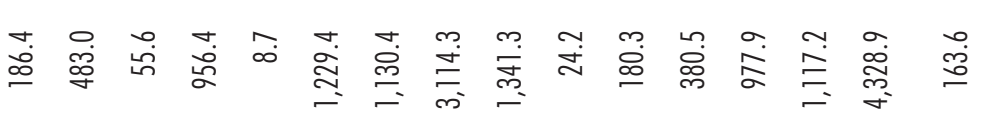

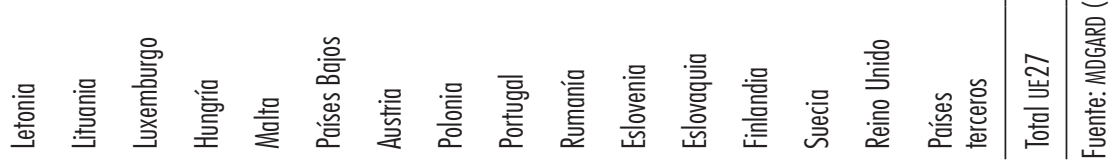


Los pagos directos de la PAC durante la segunda mitad del Marco Financiero 2007-2013 aportan dos informaciones adicionales. Una es que la agricultura europea continúa estando fuertemente subvencionada, ¿cómo refutarlo cuando casi un tercio de su renta deriva de las subvenciones? Este neoproteccionismo no es patrimonio de la UE, las ayudas a la agricultura son una práctica bastante extendida, aun en los países en vías de desarrollo. En las economías prósperas, las subvenciones de Japón, Suiza y Noruega alcanzan cuotas más elevadas; las de Estados Unidos y Canadá tampoco son escasas, aunque menores que las europeas.

La segunda información es que dichos pagos suponen una transferencia de recursos desde la industria y los servicios a la agricultura, esto contribuye, en el interior de los Estados, a reducir considerablemente las desigualdades de renta entre el sector agrario y el resto de las actividades económicas, así como entre el medio urbano y el rural. El hecho de que la mayor parte de la tierra cultivada de los países atrasados arroje un cociente de pagos notablemente inferior al de países ricos, explicita que la PAC favorece en mayor medida la armonización sectorial y territorial de rentas en el seno de las economías nacionales que entre agriculturas europeas, lo que no es el camino óptimo para combatir la dispersión de rentas existente en la UE27.

Son varias las conclusiones finales que pueden extraerse de lo expuesto sobre la política de cohesión y la PAC. En primer término, ambas son un mecanismo de armonización de rentas, combatiendo las profundas disparidades que existen en la UE27. La diferencia entre ellas es que, mientras los recursos de la política de cohesión se orientan, mayoritariamente, a la armonización territorial, los de la PAC tienen una tonalidad básicamente sectorial.

Otra conclusión es la desigual evolución de estas políticas. El Marco Financiero 2007-2013 confirma el proceso iniciado tras el Acta Única de 1986: crecimiento más intenso de los créditos destinados a la cohesión que los dirigidos a la PAC. Mas a pesar del elevado incremento que experimentan los recursos para la política de cohesión en el ciclo plurianual actual frente al precedente (2000-2006), se constata un debilitamiento en relación con la etapa de la UE15. Este fenómeno contrasta con el hecho de que la dispersión de rentas en la UE27 es muy superior a la existente en la UE15.

Tanto la política de cohesión como la PAC presentan el mismo rasgo en el Marco Financiero 2007-2013: los doce países incorporados en 2004 y 2007 no salen, paradójicamente, favorecidos en la distribución de recursos. Por el contrario, son menores las cantidades que reciben en ambas políticas, entra en contradicción con uno de los principios que ha vertebrado la UE: la solidaridad; aun con todo, dichos fondos están contribuyendo significati- 
vamente al desarrollo de estos países. En consecuencia, uno de los retos del Presupuesto 2014-2020 es remodelar y reforzar la dimensión armonizadora de la política de cohesión y de la PAC, desafío que recoge la Estrategia Europa 2020.

\section{BIBLIOGRAFÍA}

Barca, F., Un programa de reforma de la política de cohesión, Luxemburgo, 2011. Comisión Europea, Libro verde sobre la cohesión territorial. Convertir la diversidad territorial en un punto fuerte [Сом (2008) 616 final], Luxemburgo, 2008.

Comisión Europea, Quinto informe de situación sobre la cohesión económica y social. Las regiones crecen y Europa crece, [сом (2008) 371], Luxemburgo, 2008, 371.

Comisión Europea, Una PAC simplificada para Europa: un beneficio para todos, Luxemburgo, 2009.

Comisión Europea, Conclusiones del V Informe sobre la cohesión económica, social y territorial: el futuro de la política de cohesión [СОм (2010) $642 \mathrm{final}$ ], Luxemburgo, 2010.

Comisión Europea, La PAC en el horizonte de 2020: Responder a los retos futuros en el ámbito territorial, de los recursos naturales y alimentarios [СОм (2010) 672 final], Luxemburgo, 2010.

Comisión Europea, Revisión del presupuesto de la UE [сом (2010) 700], Luxemburgo, 2010.

Comisión Europea, Cohesion Policy 2014-2020, Investing in Growth and Jobs, Luxemburgo, 2011.

Comisión Europea, Propuesta de la Comisión Europea de Marco Financiero Plurianual 2014-2020, Luxemburgo, 2011.

Comisión Europea, Un presupuesto para Europa 2020 [sEC (2011) 867 final y SEC (2011), 868 final], Luxemburgo, 2011.

Comisión Europea, Presupuesto General de la Unión Europea. Resumen de datos, Luxemburgo, varios años.

Cooper, T., K. Hart, y Baldock, Provision of Public Goods Through Agriculture in the European Union, Institute for European Environmental Policy, 2009.

DG Agriculture and Rural Development, "Why do we Need a Common Agriculture Policy?”, Discussion paper (dic.), Luxemburgo, 2009.

DG Agriculture and Rural Development, Agricultural Statistics. Main Results, 2008, 2009, Luxemburgo, 2010. 
DG Agriculture and Rural Development (2010), Rapport Agricola Annual, Luxemburgo.

DG Agriculture and Rural Development, Rural Development in the European Union. Statistical and Economic Information Report, Luxemburgo, 2010.

DG Agriculture and Rural Development, Agriculture in the European Union. Statistical and Economic Information Report 2010, Luxemburgo, 2011.

Gorton, M. y C. Hubbard, "The Folly of European Union Policy Transfer: Why the Common Agricultural Policy (CAP) does not Fit Central and Eastern Europe", Regional Studies, Taylor Francis, vol. 43, 2009.

González Temprano, A., "El debilitamiento de la política de cohesión de la Unión Europea, 2007-2010”, Presupuesto y Gasto Público, núm. 66, 2012. Rawson, J. M., "Agricultural Research, Education, and Extension: Issues and Background", Congressional Research Service, The Library of Congress, marzo, 2006.

Sapir, A., An Agenda for a Growing Europe. Making the EU Economic System Deliver, Luxemburgo, 2003. 